Craniofacial and Skeletal Diseases Branch, National Institute of Dental and Craniofacial Research, National Institutes of Health, Building 30, Room 228, 30 Convent Drive MSC 4320, Bethesda, Maryland 20892, USA. Phone: (301) 496-4563; Fax: (301) 402-0824; E-mail: PROBEY@dir.nidcr.nih.gov.

The concept of a stem cell, now nearing its hundredth year as one of the organizing principles of developmental biology, shows no sign of losing its youthful luster. At a time when the transfer of biological concepts to clinical practice drives much of science, the properties of stem cells in various tissues are attracting increasing levels of interest. This attention is not restricted to the germ line and blood, the traditional domain of stem cells as defined by early 20th-century studies on animal development, but has been extended to tissues not typically thought to turn over. As novel sites, properties, and functions have been identified for these cells, the definition of a stem cell has shifted repeatedly. Like some stem cells, this concept has expanded greatly and has displayed a remarkable degree of plasticity.

\section{The origins of the concept}

The recognition that tissues vary in their capacity to regenerate and the identification of tissues that can selfrenew over an organism's complete life span are rooted in 19th-century biological and medical science. The existence of a stem cell, viewed as the ultimate origin of selfrenewal in self-renewing tissues, was perhaps first postulated by Regaud based on his studies of spermatogenesis $(1,2)$. Concurrently, the hematologists Weidenreich, Dantschakoff, and Maximow provoked a hot debate, sustained by Ferrata and Pappenheim, proposing that all blood cells derive from a common stem cell (reviewed in ref. 3). These astute observers, without the advantages of current technologies, recognized that for spermatogenesis to occur or for blood cells to be replenished throughout the lifespan of the organism, there must be a self-renewing ancestral cell. Thus, historically, the concepts of stem cells and tissue self-renewal became closely linked (4). Later, the establishment of appropriate hematological assays (5) and the finding that the progeny of certain clonogenic progenitors could completely reconstitute hematopoiesis following lethal irradiation more clearly delineated the role of the stem cell, providing the basis for the most conspicuous translation to date of stem cell biology into medical treatment: bone marrow transplantation. Consequently, the ability of a stem cell to reconstitute a dependent tissue for the lifespan of an organism also became a part of the generic definition of a cell's "stemness."

Both of these "classical" facets of a stem cell's character, self-renewal and tissue reconstitution, are currently being challenged. Today, as discussed in several articles in this Perspective series (6-8), tissues typically regard- ed as non-self-renewing (such as nervous tissue), slowly self-renewing (such as bone), or non-self-renewing but endowed with a limited capacity to repair (such as skeletal muscle) are all said to include stem cells. However, in all of these cases, there is no clear evidence such as exists for stem cells in the hematopoietic system - that the putative stem cells undergo self-renewal in vivo. In most cases, progenitor cells, which have sometimes been presumed equivalent to endogenous stem cells, can be isolated and expanded extensively, allowing for impressive, but not necessarily unlimited, amounts of the dependent tissues to be generated.

The implicit linkage of unlimited self-renewal in vivo to extensive expansion ex vivo represents a remarkable and often unnoticed shift in thinking. This shift merits careful scrutiny, however, since it invites us to define the stem cell on the basis of a biological property that exists only under experimental conditions. Likewise, clonogenicity, or the ability of a single cell to proliferate independently to form a colony, is another property commonly ascribed to stem cells, although many clonogenic cells are limited in their capacity for expansion ex vivo. As with expandability, clonogenicity is defined solely on the basis of an ex vivo assay. Thus, we have learned to recognize stem cells, not necessarily by what they do in their dependent tissues within an organism, but rather by what we can do with them in the laboratory. These two parameters, ex vivo expansion and clonogenicity, have provided us the novel perspective that even tissues that never apparently turn over have stem cells, a remarkable advance in our understanding.

\section{Potency and plasticity}

In traditional thinking, stem cells have been generally recognized as undifferentiated cells with varying degrees of potency - a measure of the number and range of phenotypes that they can develop into. Studies of cell populations during embryonic development have lead to the identification of cells that are totipotent, that is, a single cell can by itself give rise to an entire organism. However, totipotency is fleeting, disappearing after the early divisions of a fertilized oocyte, but reappearing after formation of the inner cell mass, which contains embryonic stem (ES) cells that can participate in forming every tissue of the organism. However, they are only totipotent within an environmental context, either during development or upon transplantation into a postnatal organism; a single ES cell, unlike a zygote, cannot form a complete organism by itself. As 
development proceeds, other stem cells emerge with more limited proliferative and differentiative capabilities. Each new cell formed becomes progressively more and more restricted, based on the reactions of its ancestors to a changing environment (9), and following the general rule that differentiation is accompanied by decreased proliferation (10).

Postnatally, the distinction between progenitors and stem cells becomes somewhat unclear, because of our changing views of what defines these cells. Progenitor cells associated with postnatal somatic tissues are generally seen as committed cells left over from development and, as such, would be expected to be capable of a limited number of divisions and to generate differentiated cells of only the phenotypes expected for a given tissue. Stem cells, conversely, should be capable of sustained renewal and tissue regeneration. Such postnatal cells may be unipotent, giving rise to a singular phenotype, or pluripotent where progeny have multiple distinct phenotypes. By this standard, pluripotency of the kind seen in hematopoietic stem cells (HSCs) is not an obligatory property of stem cells, but it is plausibly seen as being limited to them. It is generally assumed that the mechanisms of lineage restriction observed during development apply to the progeny of postnatal stem cells. However, development is a system of differentiation, not renewal, and it is not certain that all stem cells found in the postnatal organism are governed by the same principles that dictate stem cell activity during development. Nevertheless, some postnatal stem cells clearly behave like developmental stem cells, since a decrease in proliferation, coupled with a progressive commitment and lineage restriction, governs the formation of blood cells from HSCs in adult animals.

In contrast to the pluripotent nature of the HSC, the fates of its progeny are restricted. Thus, a monocyte may become an alveolar macrophage or a Kupffer cell, but it will never become an erythrocyte. However, such restrictions do not apply in other tissues, where differentiated cells display plasticity, the ability to alter their phenotypes in response to changes in their local environment. This property was first noted in studies on skeletal stem cells found in the bone marrow stroma, where shifts in phenotypes of even differentiated cells were observed earlier both in vivo and in vitro (see Bianco and Robey, this Perspective series, ref. 7). Furthermore, in some tissues, more differentiated cells can give rise to stem cells and may even replenish the stem cell population (see Booth and Potten, this Perspective series, ref. 11). The need for nonplastic and plastic cells may derive from the economics of tissue homeostasis. In tissues where rapid renewal is essential, such as blood, skin, and intestine, it is far more efficient to continuously generate a series of cells with dedicated phenotype (4); conversely, in tissues that turn over more slowly, such as hard and soft connective tissues, plasticity is essential to accommodate growth and response to injury (12).

Just as there are degrees of potency among stem cells, both they and their progeny display degrees of plasticity. As shown in the bone marrow stromal system, differentiated cells shift between osteoblastic cells, myelosupportive stroma, and adipocytes, within the context of the bone/marrow organ environment (see refs. 7, 12). However, striking experimental observations indicate there is also plasticity at the stem cell level. For example, neural and muscle stem cells can give rise to blood, and marrow stroma-derived stem cells can generate neural tissue (see Perspectives in this series, refs. 6-8). Some stem cells thus display a higher level of plasticity upon removal from their normal confines, when they take on the phenotypes of cells indigenous to the new context in which they are placed. This lack of cellular fidelity to a specific tissue or lineage violates classical expectations about developmental pathways. Perhaps, however, it should no longer surprise us after the recent spectacular demonstrations that a nucleus transplanted from a differentiated somatic tissue can contribute to all the cells of a complete organism. The cloning of a whole animal, as was seen with the sheep, Dolly (13), and a number of animals of several mammalian species, clearly calls into question the notion that differentiation is terminal, although it remains unclear whether adult cells that maintain their plasticity are in some way exceptional. In fact, the majority of nuclear transfers are unsuccessful, and there are no criteria for predicting which nuclei can be used to generate a totipotent cell. These findings may indicate that stem cells with embryonic character persist throughout life, whether they are actually used in tissue turnover or not. Plasticity of differentiated cells and stem cells has become a new frontier of cell biology. Understanding to what extent the two types of plasticity exist, and in what tissues, in what cells, at what ages, is a major challenge to the field.

\section{Nature and nurture}

The role of environmental cues, as opposed to genetic determination, in the specification of a cell's fate has long been debated. More than two decades ago (14), before molecular descriptions of gene regulation or an understanding of cell-environment interactions were developed, a remarkable flavor of a "Darwinism vs. Lamarckism" contest was attached to the debate. We now know that environmental cues play a central role in cell differentiation during development, postnatal growth, and maintenance of cell populations in a tissue. Postnatal stem cells are extremely sensitive to the physical nature of their environments. Even HSCs are initially regulated in such fashion through their interaction with cells of the bone marrow stroma, but after being induced to proliferate and differentiate, hematopoietic cells are solitary. Their further differentiation occurs in a predominantly fluid, matrix-free environment and is driven by soluble factors and the intrinsic character of the cells themselves. In solid tissues, conversely, cell-cell and cell-matrix interactions exert powerful and continuous effects, not only in directing a cell's development and behavior, but also in maintaining its state of differentiation. The significance of these environmental cues is further confirmed by the recent unexpected finding that altering the physical location of certain stem cells causes a complete reprogramming of their phenotypic expression patterns. 
The plasticity of differentiated cells and stem cells in the postnatal organism poses important questions concerning the role of environmental cues. What mechanisms allow a stem cell to escape developmental pressures and maintain its "stemness"? What macro- or microenvironmental cues maintain a cell in its differentiated state? If cells associated with adult tissues and organs retain the ability to differentiate along both orthodox (tissue- or lineage-restricted) and unorthodox (unrestricted) pathways, what specific environmental cues do they respond to? If we can determine these mechanisms and cues, can we alter them to our benefit, to stimulate stem cell recruitment, proliferation, and induction into a desired phenotype in vivo (see Cossu and Mavilio, this Perspective series, ref. 8)? Other important questions relate to the developmental origin of postnatal stem cells and their possible relationships, as well as the role of symmetrical and asymmetrical cell divisions that maintain stem cell compartments but allow for differentiation (Booth and Potten, this Perspective series, ref. 11; Morris, this series, ref. 15).

\section{Stem cells, regeneration, and disease}

The recognition that somatic stem cells can be isolated and are able to renew a particular tissue motivated immediate efforts to apply these cells in the clinic. Bone marrow transplantation, albeit not successful in all circumstances, has become a mainstay in the treatment of hematological and some nonhematological diseases and cancers (16). Extensive skin lesions are now being treated with the use of autologous and even nonautologous grafts generated by the ex vivo expansion of epidermal cells (17). The reconstruction of damaged articular cartilage has been attempted using ex vivo expanded chondrogenic cells (18). More recently, it has also been suggested that skeletal tissue, muscle, and even nervous tissue can be regenerated from stem cell populations (discussed in refs. 6-8). Potential applications extend beyond tissue regeneration, into the realm of gene transfer and gene therapy. With the advance of molecular techniques, it is envisioned that stem cells could be engineered to replace or repair a defective gene. Because of their self-renewal and ability to regenerate a tissue, transgenic stem cells could provide a long-lasting clinical benefit to a recipient. Although the precise techniques for accomplishing these goals are not yet in hand, our biotechnological imaginations have run wild with the hope of recreating organs, correcting genetic diseases, and improving the quality of life as we age.

The realization that cells with extensive potential for growth and differentiation occur in a variety of tissues also provides novel angles for understanding disease mechanisms. Since stem cells regulate the dynamics of normal tissues, a surprising range of disorders, including gastric atrophy, Alzheimer's disease, and, perhaps more intuitively, various forms of cancer (for example, see Morris, this Perspective series, ref. 15), can be traced to altered stem cell function. The trend toward defining stem cells primarily based on our ability to manipulate them in culture should also inspire us to devise novel models of these diseases, by analyzing genetically altered or carcinogen-treated stem cells either in vitro or in vivo after transplantation into host animals. Thus, even without improved tissue engineering or replacement, the study of stem cells may deepen our understanding of their pathogenic roles and facilitate the design of novel treatments.

This Perspective series will present many of these new aspects of stem cell biology and will show the diversity of stem cells that can be found in various nonhematopoietic tissues. The articles represent current thoughts on a broad array of stem cell types, and the topics were selected not only to draw attention to their diversity, but also to offer up for discussion to the readership at large the current controversies and challenges that face the field. As put forth in these articles, a new conceptual framework is needed for thinking about stem cells and their capacities. This framework will need to accommodate emerging findings on different classes of stem cells and should allow us to better recognize the normal and pathological roles of stem cells and to develop novel approaches for the treatment of defects and disease.

\section{Acknowledgments}

The editor of this Perspective series would like to thank Paolo Bianco (Universitá dell'Aquila, Italy) for his support and contributions to this overview.

1. Regaud, C. 1901. Etudes sur la structure des tubes seminiferes et sur la spermatogenese chez les mammiferes. Part 1. Archives d'Anatomie microscopiques et de Morphologie experimentale. 4:101-156.

2. Regaud, C. 1901. Etudes sur la structure des tubes seminiferes et sur la spermatogenese chez les mammiferes. Part 2. Archives d'Anatomie microscopiques et de Morphologie experimentale. 4:231-280.

3. Baserga, A., and Zavagli, G. 1981. Ferrata's stem cells: an historical review on hemocytoblasts and hemohistioblasts. Blood Cells. 7:537-545.

4. Leblond, C.P. 1964. Classification of cell populations on the basis of their proliferative behaviour. Natl. Cancer Inst. Monogr. 14:119-150.

5. Till, J.E., and McCulloch, E.A. 1961. A direct measurement of the radiation sensitivity of normal mouse bone marrow cells. Radiat. Res. 14:1419-1430.

6. Reference deleted in proof.

7. Bianco, P., and Robey, P.G. 2000. Marrow stromal stem cells. J. Clin. Invest. In press.

8. Cossu, G., and Mavilio, F. 2000. Myogenic stem cells for the therapy of primary myopathies: wishful thinking or therapeutic perspective? J. Clin. Invest. In press.

9. Papaioannou, V.E., Rossant, J., and Gardner, R. 1978. Stem cells in early mammalian development. In Stem cells and tissue homeostasis. B.I. Lord, C.S. Potten, and R.J. Cole, editors. Cambridge University Press. New York, New York, USA. 49-70.

10. Rusch, H.P. 1954. Carcinogenesis: a facet of living processes. Cancer Res. 14:407-417.

11. Booth, C., and Potten, C.S. 2000. Gut instincts: thoughts on intestinal epithelial stem cells. J. Clin. Invest. 105:1493-1499

12. Bianco, P., Riminucci, M., Kuznetsov, S., and Robey, P.G. 1999. Multipotential cells in the bone marrow stroma: regulation in the context of organ physiology. Crit. Rev. Eukaryot. Gene Expr. 9:159-173.

13. Wilmut, I., Schnieke, A.E., McWhir, J., Kind, A.J., and Campbell, K.H. 1997. Viable offspring derived from fetal and adult mammalian cells. Nature. 385:810-813.

14. Holtzer, H. 1978. Cell lineages, stem cells and the "quantal" cell cycle concept. In Stem cells and tissue homeostasis. B.I. Lord, C.S. Potten, and R.J. Cole, editors. Cambridge University Press. New York, New York, USA. 1-28.

15. Morris, R. 2000. Keratinocyte stem cells: targets for cutaneous carcinogens. J. Clin. Invest. In press.

16. Treleaven, J., and Barrett, J. 1992. Introduction to bone marrow transplantation. In Bone marrow transplantation in practice. J. Treleaven and J. Barrett, editors. Churchill Livingstone. New York, New York, USA. 3-9.

17. Green, H. 1989. Regeneration of the skin after grafting of epidermal cultures. Lab. Invest. 60:583-584.

18. Brittberg, M., et al. 1994. Treatment of deep cartilage defects in the knee with autologous chondrocyte transplantation. N. Engl.J. Med. 331:889-895. 